\title{
Efficacy of Herbicides against Weeds in Transplanted Wet Season Rice (Oryza Sativa L.)
}

\author{
T. Das ${ }^{1 *}$, M. Banerjee ${ }^{2}$, G. C. Malik ${ }^{3}$, B. Mandal ${ }^{4}$ \\ ${ }^{1,4}$ Department of Agriculture, Government of West Bengal, \\ ${ }^{2,3}$ Department of ASEPAN, Palli Siksha Bhavana (Institute of Agriculture), Visva-Bharati, Sriniketan- 731236. \\ West Bengal
}

\begin{abstract}
Comparative efficacy of different herbicides against weeds in transplanted kharif rice was studied at Agricultural Farm of Institute of Agriculture, Visva Bharati,Sriniketan, West Bengal. The experiment was laid out in a randomized block design with 9 treatments and 3 replications. The results revealed that the major weed flora associated with the transplanted rice during kharif season was mainly comprised of Echinochloa colona, Cyperus iria L., Sphenoclea zeylanica Gaertn. and Ludwigia parvifloraRoxb. Bispyribac sodium @30 g a.i ha${ }^{1}$ applied at 25 days after transplanting was most effective to check all types of weed population and their growth. This treatment also gave the maximum grain yield (5549 $\left.\mathrm{kg} \mathrm{ha}^{-1}\right)$ and straw yield $\left(5991 \mathrm{kgha}^{-1}\right)$ of rice resulting in lowest weed index (5.61\%) among chemical herbicide treated plots. The highest grain and straw yield value as observed with application of bispyribac sodium @ $30 \mathrm{~g}$ a.i ha $\mathrm{h}^{-1}$ was statistically at par with that of bispyribac sodium @ $25 \mathrm{~g}$ a.i ha ${ }^{-1}$ at 25 DAT. Therefore, bispyribac sodium @ $25 \mathrm{~g}$ a.i ha ${ }^{-1}$ applied at 25 days after transplanting may be recommended for weed management in transplanted kharif rice.
\end{abstract}

Key Words: Bispyribac sodium, grain yield, transplanted rice.

\section{Introduction}

Rice is the principal crop during rainy season in the plains of West Bengal. The productivity of wet season rice is very low as weeds pose serious menace as compared to other rice ecosystems. This is because of aerobic soil condition, high temperature and dry tillage practice. With the introduction of short statured high yielding rice varieties with erectophylic leaves, the weed menace is becoming more acute (Mishra et.al. 2006). The weed flora under transplanted condition is very much diverse and consists of grasses, sedges and broad-leaf weeds causing yield reduction of rice crop up to $76 \%$ (Singh et al. 2004). The effective control of weeds at initial stages (0-40 DAT) can help in improving the productivity of this crop. Therefore, evaluation of new herbicides for control of wide spectrum of weed flora is imperative. Recent trend of herbicide use is to find out an effective weed control measure by using low dose high efficiency herbicides which will not only reduce the total volume of herbicide use but also the application become easier and economic (Kathiresan, 2001). In view of the above facts, the present study was undertaken to evaluate the performance of bispyribac sodium $10 \mathrm{WP}$ in transplanted kharif rice and associated weeds.

\section{Materials And Methods}

A field experiment was carried out in the wet season of 2014 in red and lateritic zone of West Bengal at agricultural farm, Sriniketan, Birbhum under Institute of Agriculture, Visva-Bharati. The farm where the experiment was conducted is situated at red and lateritic zone of West Bengal at The field is situated at $23^{\circ}$ $40.167^{\prime} \mathrm{N}$ latitude and $087^{\circ} 39.492^{\prime} \mathrm{E}$ longitudes with an average altitude of $58.9 \mathrm{~m}$ above mean sea level. The experimental soil was sandy loam in texture having pH 5.09, EC $0.32 \mathrm{ds} \mathrm{m}^{-1}$, organic carbon $0.37 \%$, available $\mathrm{N}$, $157.93 \mathrm{~kg} \mathrm{ha}^{-1}$, available $\mathrm{P} 27.58 \mathrm{~kg} \mathrm{ha}^{-1}$ and available $\mathrm{K} 126.41 \mathrm{~kg} \mathrm{ha}^{-1}$. The experiment was laid out in a randomized block design with 9 treatments (Table 1) and 3 replications. 21 days old seedlings of rice var. MTU 1010 was transplanted with 3-4 seedlings hill ${ }^{-1}$ during 2 ndweek of August in the year of experimentation at a spacing of $20 \mathrm{~cm} \times 20 \mathrm{~cm}$. Herbicides were sprayed using knapsack sprayer fitted with a flat fan nozzle at a spray volume of $5001 \mathrm{ha}^{-1}$. Recommended dose of fertilizers i.e. 80: 40: $40 \mathrm{~kg} \quad \mathrm{~N}, \mathrm{P}_{2} \mathrm{O}_{5}$ and $\mathrm{K}_{2} \mathrm{O}$ ha $^{-1}$ were applied. Half dose of $\mathrm{N}$ in the form of Urea, and full amount of $\mathrm{P}_{2} \mathrm{O}_{5}$ (Single Super Phosphate) and $\mathrm{K}_{2} \mathrm{O}$ (Muriate of Potash) were applied as basal during final land preparation. Rest half of $\mathrm{N}$ was topdressed in two equal splits; one at active tillering and the other at panicle initiation stage. The rice plant was harvested on 31.10.2014 experimentation. The performance of different treatments was studied in terms of all types of flora, weed density, weed biomass and their subsequent effect on growth and yield of rice. 
Efficacy Of Herbicides Against Weeds In Transplanted Wet Season Rice (Oryza Sativa L.)

Table 1 Details of treatments

\begin{tabular}{|c|c|c|c|}
\hline SI. No. & Treatments & Dose (g a.i. ha $\left.{ }^{-1}\right)$ & Time of application (DAT) \\
\hline $\mathrm{T}_{1}$ & Bispyribac sodium & 20 & 25 \\
\hline $\mathrm{T}_{2}$ & Bispyribac sodium & 25 & 25 \\
\hline $\mathrm{T}_{3}$ & Bispyribac sodium & 30 & 25 \\
\hline $\mathrm{T}_{4}$ & Cyhalofop-butyl fb2,4-D & $90 \mathrm{fb} 800$ & $15 \mathrm{fb} 30$ \\
\hline $\mathrm{T}_{5}$ & Cyhalofop-butyl fb MSM+ CME & $90 \mathrm{fb} 4$ & $15 \mathrm{fb} 30$ \\
\hline $\mathrm{T}_{6}$ & Butachlor fb2,4-D & $1500 \mathrm{fb} 800$ & $3 \mathrm{fb} 30$ \\
\hline $\mathrm{T}_{7}$ & Butachlor fb MSM+ CME & $1500 \mathrm{fb} 4$ & $3 \mathrm{fb} 30$ \\
\hline $\mathrm{T}_{8}$ & Hand weeding twice & - & 20 and 40 DAT \\
\hline $\mathrm{T}_{9}$ & Weedy check & - & - \\
\hline
\end{tabular}

$\mathrm{fb}=$ followed by, DAT = Days after transplanting, MSM= metsulphuron- methyl, CME= chlorimuron- ethyl.

Effect on weeds

\section{Results And Discussion}

Among sedges,Cyperus iria L.,Fimbristylis miliacea L., among grasses Cyanodon dactylon Pers., Echinochloa crusgalli (L.) Beauv, and among broadleaved weeds Ludwigia parviflora Roxb , Marsilea quadrifolia L., Sphenoclea zeylanica Gaertn. and Alternanthera sessilis L. DC.were found dominant in the experimental plot during the experimentation. Density and biomass of weeds were significantly higher in nonweeded control treatment. In contrast, hand weeding (Twice at 20 \& 40 DAT) treatment recorded lower weed density and biomass of weeds than rest of the weed management practices (Table 2). Among the tested herbicides, bispyribac-sodium $10 \mathrm{WP}$ at $30 \mathrm{~g}$ a.i. ha $^{-1}$ applied at 25 days after transplanting (DAT) was most effective to check all types of weed population and their growth resulting in lowest biomass of weeds due to its higher weed control efficiency. The values of the same were statistically at par with that of bispyribac-sodium $10 \mathrm{WP}$ at $25 \mathrm{~g}$ a.i. ha ${ }^{-1}$ applied at 25 days after transplanting (DAT).

Effect on crop

Perusal of the Table 3 revealed that all the herbicide treated plots produced grain and straw yields significantly more than the non-weeded plots. The highest grain yield of rice $\left(6143 \mathrm{~kg} \mathrm{ha}^{-1}\right) \mathrm{was}$ obtained with hand weeding twice at 20 \& 40 DAT. Among chemical herbicide treated plotsbispyribac-sodium $10 \mathrm{WP}$ at $30 \mathrm{~g}$ a.i. $\mathrm{ha}^{-1}$ applied at 25 days after transplanting (DAT) recorded highest rice grain yield (5549 $\mathrm{kg}$ $\mathrm{ha}^{-1}$ )and it was at par with bispyribac-sodium $10 \mathrm{WP}$ at $25 \mathrm{~g}$ a.i. ha ${ }^{-1}$ applied at 25 days after transplanting (DAT) $\left(5176 \mathrm{~kg} \mathrm{ha}^{-1}\right)$. Similar trend of result wasalso found in case of straw yield of rice. The findings are in line with Manjunatha et al., (2012. The effective control of weedsstarting from the early crop growth stage might have resultedin better growth and yield of rice. The variation in grain yieldunder different treatments was the result of variation in weeddensity and weed biomass. Application of herbicides undertest did not show any phytotoxic symptom on rice plant.

Table 2 Effect of different treatments on weed density, weed biomass and weed control efficiency on rice

\begin{tabular}{|c|c|c|c|c|c|c|}
\hline \multirow[t]{2}{*}{ Treatments } & \multicolumn{2}{|c|}{ Total weed Density $\left(\mathrm{m}^{-2}\right)$} & \multicolumn{2}{|c|}{$\begin{array}{llll}\begin{array}{l}\text { Total } \\
\left(\mathrm{gm}^{-2}\right)\end{array} & \text { weed bio } & \text { mass } \\
\end{array}$} & \multicolumn{2}{|c|}{$\begin{array}{l}\text { Weed control } \\
\text { efficiency }(\%)\end{array}$} \\
\hline & 40 DAT & 60 DAT & 40 DAT & 60 DAT & 40 DAT & $60 \mathrm{DAT}$ \\
\hline $\begin{array}{l}\mathrm{T}_{1} \text { :- Bispyribac-sodium @ } 20 \mathrm{~g} \text { a.i./ ha at } 25 \\
\text { DAT }\end{array}$ & $4.90(23.50)^{*}$ & $5.72(32.25)$ & $1.39(1.44)$ & $2.28(4.70)$ & 93.58 & 88.71 \\
\hline $\mathrm{T}_{2}:-$ Bispyribac-sodium @ $25 \mathrm{~g}$ a.i./ ha at $25 \mathrm{DAT}$ & $4.50(19.72)$ & $4.90(23.50)$ & $1.30(1.19)$ & $2.20(4.35)$ & 94.67 & 89.56 \\
\hline $\begin{array}{l}\mathrm{T}_{3}:- \text { Bispyribac-sodium @ } 30 \mathrm{~g} \text { a.i./ ha at } 25 \\
\text { DAT }\end{array}$ & $3.97(15.24)$ & $4.50(19.72)$ & $1.17(0.88)$ & $2.08(3.81)$ & 96.08 & 90.84 \\
\hline $\begin{array}{l}\mathrm{T}_{4}:-\mathrm{CFB} @ 90 \mathrm{~g} \text { a.i./ha at } 15 \mathrm{DAT} \text { fb 2,4-D-Na@ } \\
0.8 \mathrm{~kg} \text { a.i./ha at } 30 \mathrm{DAT}\end{array}$ & $6.56(42.55)$ & $6.56(42.55)$ & $1.68(2.31)$ & $2.61(6.32)$ & 91.84 & 86.21 \\
\hline $\begin{array}{l}\mathrm{T}_{5}:-\mathrm{CFB} @ 90 \mathrm{~g} \text { a.i./ha at } 15 \text { DAT fb } \\
\text { CME (Almix) @ 4g a.i./ha at 30 DAT }\end{array}$ & $5.75(32.51)$ & $5.75(32.51)$ & $1.52(1.82)$ & $2.50(5.74)$ & 89.69 & 84.83 \\
\hline $\begin{array}{l}\mathrm{T}_{6}:- \text { Butachlor @ } 1.5 \mathrm{~kg} \text { a.i./ha at } 3 \text { DAT fb 2,4-D- } \\
\mathrm{Na} @ 0.8 \mathrm{~kg} \text { a.i./ha at } 30 \mathrm{DAT}\end{array}$ & $7.88(61.52)$ & $7.88(61.52)$ & $1.97(3.36)$ & $3.08(9.02)$ & 89.09 & 81.91 \\
\hline $\begin{array}{l}\mathrm{T}_{7}:- \text { Butachlor @ } 1.5 \mathrm{~kg} \text { a.i./ha at } 3 \text { DAT fb MSM + } \\
\text { CME (Almix) @ 4g a.i./ha at 30 DAT }\end{array}$ & $7.02(48.83)$ & $7.02(48.83)$ & $1.71(2.42)$ & $2.83(7.53)$ & 84.96 & 78.31 \\
\hline $\mathrm{T}_{8}$ :- Hand weeding at 20,40 DAT & $0.71(0.00)$ & $3.97(15.24)$ & $0.71(0.00)$ & $1.19(0.91)$ & 100.00 & 97.82 \\
\hline $\mathrm{T}_{9} \quad$ :- Weedy check & $9.51(89.85)$ & $9.51(89.85)$ & $4.77(22.30)$ & $6.49(41.58)$ & - & - \\
\hline $\operatorname{LSD}(\mathrm{P}=0.05)$ & 0.86 & 0.86 & 0.25 & 0.19 & NA & NA \\
\hline
\end{tabular}

*values in parentheses are original. Data transformed to square root transformation. $\mathrm{fb}=$ followed by, $\mathrm{DAT}=$ Days after transplanting. $\mathrm{CFB}=$ cyhalofop-butyl, $\mathrm{MSM}=$ metsulphuron- methyl, $\mathrm{CME}=$ chlorimuron- ethyl, $\mathrm{NA}=$ Not analyzed 
Table 3 Effect of different treatments on yield of rice

\begin{tabular}{|c|c|c|c|}
\hline Treatments & Grain $\left(\mathrm{Kg} \mathrm{ha}^{-1}\right)$ & $\operatorname{Straw}\left(\mathrm{Kg} \mathrm{ha}^{-1}\right)$ & Weed index $(\%)$ \\
\hline $\mathrm{T}_{1}$ :- Bispyribac-sodium @ $20 \mathrm{~g}$ a.i./ ha at $25 \mathrm{DAT}$ & 4998 & 5708 & 13.49 \\
\hline $\mathrm{T}_{2}$ :- Bispyribac-sodium @ $25 \mathrm{~g}$ a.i./ ha at $25 \mathrm{DAT}$ & 5176 & 5863 & 10.39 \\
\hline $\mathrm{T}_{3}$ :- Bispyribac-sodium @ $30 \mathrm{~g}$ a.i./ ha at $25 \mathrm{DAT}$ & 5549 & 5991 & 3.94 \\
\hline $\begin{array}{l}\text { T4:-CFB @ } 90 \mathrm{~g} \text { a.i./ha at } 15 \text { DAT fb 2,4-D-Na@ } 0.8 \mathrm{~kg} \text { a.i./ha } \\
\text { at } 30 \text { DAT }\end{array}$ & 3772 & 4733 & 24.25 \\
\hline $\begin{array}{l}\mathrm{T}_{5}:-\mathrm{CFB} @ 90 \mathrm{~g} \text { a.i./ha at } 15 \mathrm{DAT} \text { fb MSM + CME (Almix) } \\
\text { @ } 4 \mathrm{~g} \text { a.i./ha at } 30 \mathrm{DAT}\end{array}$ & 4376 & 5390 & 34.71 \\
\hline $\begin{array}{l}\mathrm{T}_{6} \text { :-Butachlor @ } 1.5 \mathrm{~kg} \text { a.i./ha at } 3 \text { DAT fb 2,4-D-Na @ } 0.8 \mathrm{~kg} \\
\text { a.i./ha at } 30 \text { DAT }\end{array}$ & 3391 & 4637 & 34.97 \\
\hline $\begin{array}{l}\mathrm{T}_{7}:- \text { Butachlor @ } 1.5 \mathrm{~kg} \text { a.i./ha at } 3 \text { DAT fb MSM + CME } \\
\text { (Almix) @ 4g a.i./ha at } 30 \text { DAT }\end{array}$ & 3757 & 4683 & 41.30 \\
\hline $\mathrm{T}_{8} \quad$ :- Hand weeding at 20,40 DAT & 6143 & 6671 & - \\
\hline $\mathrm{T}_{9} \quad$ :- Weedy check & 2961 & 4386 & 48.74 \\
\hline $\operatorname{LSD}(\mathrm{P}=0.05)$ & 575.57 & 703.33 & NA \\
\hline
\end{tabular}

$\mathrm{fb}=$ followed by, DAT $=$ Days after transplanting. $\mathrm{CFB}=$ cyhalofop-butyl, $\mathrm{MSM}=$ metsulphuron- methyl, $\mathrm{CME}=$ chlorimuron- ethyl, NA= Not analyzed

Based on the results of present investigation it can be concluded that bispyribac-sodium $10 \mathrm{WP}$ at $25 \mathrm{~g}$ a.i. ha $^{-1}$ applied at 25 days after transplanting (DAT) was most effective to check all types of weed population which may be recommended to replace the tedious, time consuming and expensive hand weeding practice of weed control in transplanted wet season rice.

\section{Reference}

[1]. Singh, V. P., Singh G., Singh, M. 2004 Effect of fenoxaprop-P-ethyl on transplanted rice and associated weeds. Indian Journal of Weed Sciences 36: 190-92.

[2]. Mishra, G. C., Rath, A. K., Rath, B. S., Sahoo, J., Mohapatra, P. K. 2004. Weed management indirect seeded rice under rainfed upland eco-system. Journal of Crop and Weed 2: 52-57.

[3]. Manjunatha, K. B., Hanumanthappa, M., Nagesha, L.; Kalyanamurthy, K. N., Kamath, K. V. S. (2012). Effect of new herbicide molecules on nutrient uptake in transplanted rice (Oryzasativa L.) in coastal Karnataka. Mysore Journal of Agricultural Sciences. 46(4): 928-930 